\title{
1 Synemin-related skeletal and cardiac myopathies: an overview of pathogenic variants
}

2

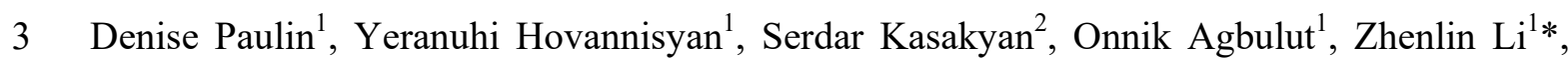
4 Zhigang Xue ${ }^{1}$

5

$6{ }^{1}$ Sorbonne Université, Institut de Biologie Paris-Seine (IBPS), CNRS UMR 8256, INSERM

7 ERL U1164, Biological Adaptation and Ageing, 75005, Paris, France.

$8 \quad 2$ Duzen Laboratories Group, Center of Genetic Diagnosis, 34394, Istanbul, Turkey.

11 Running title: Synemin polymorphism and related myopathies

*Corresponding Author:

16 Dr Zhenlin Li, Sorbonne Université, Institut de Biologie Paris-Seine, UMR CNRS 8256,

17 INSERM ERL U1164, 7, quai St Bernard - case 256 - 75005 Paris-France.

18 Phone :+33-144273421, Email : zhenlin.li@sorbonne-universite.fr 


\section{List of abbreviations}

22 AKAP, A-kinase-anchoring protein

23 IF, Intermediate filament

24 IFAPs, IF associated proteins

25 GFAP, Glial fibrillary acidic protein

26 KO, Knocked-out

27 MAF, Minor allele frequency

28 PKA, Protein Kinase A

29 SNP, Single nucleotide polymorphism

30 SRF, Serum response factor

31 


\section{Abstract}

33 This review analyzes data concerning patients with cardiomyopathies or skeletal myopathies 34 associated with a variation in the intermediate filament (IF) synemin gene (SYNM), also 35 referred to as desmuslin $(D M N)$. Molecular studies demonstrate that synemin copolymerizes 36 with desmin and vimentin IF and interacts with vinculin, $\alpha$-actinin, $\alpha$-dystrobrevin, dystrophin, 37 talin and zyxin. It has been found that synemin is an A-kinase-anchoring protein (AKAP) that anchors Protein Kinase A (PKA) and modulates the PKA-dependent phosphorylation of several cytoskeletal substrates such as desmin. As several IF proteins, including desmin, have been implicated in human genetic disorders such as dominant or recessive congenital and

41 adult onset myopathy, synemin becomes a significant candidate for cardiac and skeletal 42 myopathies of unknown etiology. Since SYNM is a new candidate gene that displays 43 numerous sequence polymorphisms, in this review, we summarize the genetic and clinical 44 literature about SYNM mutations. Protein changing variants (missense, frameshifts, nonsense) were further evaluated based on structural modifications and amino acid interactions. We present in silico modeling of helical salt-bridges between residues to evaluate the impact of

47 the synemin networks crucial to interactions with cytoskeletal proteins. Finally, a discussion 48 is featured regarding certain variants that may contribute to the disease state.

Keywords: Intermediate Filament; Cytoskeleton; Desmuslin; Desmin; polymorphism;

\section{1 genetic variant.}




\section{Introduction}

57 Intermediate filament (IF) proteins are constituents of the cytoskeleton encoded by a 58 dispersed multigene family of up to 70 genes. IFs are formed by nonglobular flexible protein 59 units, which consist mostly of $\alpha$-helices. IF protein structure comprises a central $\alpha$-helical rod 60 domain as its main part, flanked to a variable head and tail parts. IF proteins are chemically heterogeneous, have different rates of assembly and produce discrete types of filaments (16). In most vertebrate cells, cytoplasmic IFs are tethered to the nucleus. They extend into the cytoplasm providing a scaffold for mitochondria, the Golgi complex, microtubule organizing centers and other cytoskeletal elements (13). In the periphery, IFs associate with the plasma membrane. The resulting network integrates and organizes the cytoplasm providing 66 mechanical integrity, which is essential to tissue function. This process has been highlighted in more than 75 human genetic diseases. They are the result of deficiencies in this network resulting in skin fragility and epidermolytic disorders, laminopathies, myopathies, cardiomyopathies, neuropathies, cataracts and premature aging (Human Intermediate Filament Mutation Database, http://www.interfil.org).

SYNM is a new candidate gene which shows numerous sequence polymorphisms. However, few data have been available from pathologists with regards to synemin. Synemin is considered as IF associated protein (IFAPs) (15). In this respect, synemin is associated with multiple partners such as desmin, vimentin, keratins, glial fibrillary acidic protein (GFAP), neurofilaments and is present in muscle cells, in a few neural cells and in various other nonepithelial cell types. Accordingly, it can be responsible for human diseases of unknown etiology. Data are summarized here concerning patients with cardiomyopathy or skeletal myopathy in whom a variation in the SYNM gene exists. Moreover, in silico modeling of helical salt-bridges between residues is presented, allowing for the evaluation of the impact of synemin networks, which are crucial to interactions with cytoskeletal proteins. 


\section{Gene expression}

82 In humans, SYNM, also known as desmuslin $(D M N)$, was localized to chromosome $15 \mathrm{q} 26.3$

83 by electronic screening of the human DNA sequence database and using the GB4 radiation

84 hybrid panel (29). It was located in a 4-cM interval flanked by the microsatellite markers

85 D15S212 and D15S642, and close to D15S120 (48), (GRCh38:chr15:99098217-99135593).

86 Synemin was first identified as a high-molecular-mass protein associated with desmin and

87 vimentin filaments in chicken muscles (14). Synemin expression was later detected in

88 different tissues, including the nervous system, endothelial cells, retinal cells and hepatic

89 stellate cells $(18-20,41,47,50,53)$. The SYNM gene encodes three isoforms in mice and

90 humans via an alternative splicing mechanism giving rise to three isoforms: high $(\mathrm{H})$ or $\alpha$

91 (170 kDa), middle (M) or $\beta(140 \mathrm{kDa})$, and low (L) (41 kDa) synemin with the same head and

92 rod domains but differing in the tail domain of the protein (see Figure 1) $(29,48,53)$. SYNM

93 expression is regulated in an isoform specific manner during the development and

94 regeneration of different tissues.

95 During mouse embryo development, synemin $\mathrm{M}$ was present as early as in 5-day-old embryo

96 (E5) of mice with vimentin and nestin. Synemin H was found later at E9 in the nervous

97 system and mesodermic derivatives concomitantly with angiogenesis and the migration of

98 neural crest cells. Synemin L appeared later in neurons at E15 (20). Moreover, de Souza

99 Martins et al. demonstrated that synemin $\mathrm{M}$ is expressed in mouse pluripotent embryonic

100 stem cells while synemin $\mathrm{H}$ was produced only in neural precursors when neural

101 differentiation began, concurrently with synemin $\mathrm{M}$, nestin and glial fibrillary acidic protein

102 (GFAP) (10). The expression of synemin $\mathrm{H}$ and $\mathrm{M}$ was reported in multipotent neural stem

103 cells, particularly in the subventricular zone of the lateral ventricle of the adult brain; a

104 neurogenic germinal niche of mice (19). 
105 The expression of synemin has also been demonstrated in skeletal muscle satellite cells (26).

106 Synemin M was present in all types of smooth muscles (blood vessels, bladder, and stomach)

107 whereas synemin $\mathrm{H}$ and L were detected solely in the bladder (53). Furthermore, a study

108 identified synemin as a novel serum response factor (SRF)-independent target of myocardin

109 that is abundantly expressed in all smooth muscle cells (46). In striated muscles, the

110 predominant synemin isoforms was $\mathrm{M}$ but all three synemin isoforms were produced by an

111 alternative splicing of the synemin pre-mRNA and their expression was regulated in a

112 developmentally specific manner (53). The temporal distributions of the three synemin

113 isoforms in striated muscles were distinct. The $\mathrm{H} / \mathrm{M}$ isoforms were detected in the epaxial

114 muscles (myotomes) and the heart as early as E10 in mice, and they persisted throughout all

115 embryo stages and in the adult. In contrast, the L isoform was found only in a subset of adult

116 muscles (53). These differences probably reflect differing functions of the synemin isoforms

117 during development.

\section{Synemin interactions with other proteins}

119 Synemin consists of a short head domain, a missing conserved TALL sequence in the 2A

120 subdomain that is characteristic of all IFs, and the TYRKLLEGEE motif in the $2 \mathrm{~B}$ subdomain

121 that differs from all other IFs (24). In this respect, synemin is associated with multiple

122 partners such as desmin, vimentin, keratins, GFAP, neurofilaments to form the filament in

123 different tissues. Synemin co-assembles with desmin and vimentin to become heteropolymers

124 in striated and smooth muscle tissues (53). It interconnects Z-discs to costamere in skeletal

125 muscle (1) and to desmosomes in the heart (27) as well as participates in mitochondria

126 positioning, a process that probably involves plectin (25). The synemin protein interacts with

127 and is closely related to desmin (34); a protein encoded by a locus mutated in some forms of

128 hereditary distal myopathy (28). In myopathies caused by desmin mutation, synemin is

129 associated with cytoplasmic desmin aggregates in skeletal muscles $(4,5,7,32,34,48)$. 
130 Interestingly, in desmin mutations located into the highly conserved amino acid motif of helix

131 2B (E401K, R406W, E413K), synemin is excluded from desmin containing aggregates (7). In

132 this study, using in silico constructs the authors demonstrated that such behavior with these

133 mutants was correlated to the abnormal salt-bridges of desmin-dimer which prevent

134 longitudinal annealing and/or radial compaction during desmin filament formation. This

135 perturbs synemin-desmin heteropolymer filament assembly (7). Co-transfection and

136 immunolabeling experiments indicate that both synemin $\mathrm{H}$ and $\mathrm{M}$ isoforms are incorporated

137 to desmin to form heteropolymeric IFs. Furthermore, using desmin- and vimentin-deficient

138 mice whose synemin was unstable and delocalized in cells, there is direct evidence of

139 association with desmin or vimentin in skeletal and smooth muscles $(6,19,22,53)$. Synemin

140 also interacts with plectin, zyxin, $\alpha$-actinin, dystrophin, utrophin, $\alpha$-dystrobrevin, vinculin and

141 metavinculin, which highlights its role as a bridging protein $(2,3,17,21,25,29,33,44,45)$

142 (see Figure 2).

143 Phosphorylation of IF proteins in serine and threonine residues causes rearrangements and

144 interactions with other cellular proteins $(8,49,52)$. It has been demonstrated that synemin is

145 an A-kinase-anchoring protein (AKAP) (40) that anchors protein kinase A (PKA) and

146 modulates the PKA-dependent phosphorylation of several cytoskeletal substrates such as

147 desmin and as such, may influence muscle growth and repair by regulating the

148 phosphorylation of proteins of the Z-disks and sarcolemma $(12,26)$. In addition, synemin also

149 interacts with Akt (36); a kinase important for the normal growth of fast twitch muscles.

150 These observations collectively reinforce the idea that synemin has dual functions acting both

151 as a bridging cytoskeletal protein and as a regulator of signal transduction pathways.

\section{Loss of synemin in mice leads to cardiac and skeletal myopathies}

153 Two independent synemin deficient mice were generated using homologous recombination

154 method, one of them using the Cre-loxP mediated system to remove exon 1 (26), the second 
155 model was a constitutive knocked-out (KO) by removing all exons (12). The KO mice are

156 viable, fertile and were born at expected Mendelian ratio. Synemin is not required for 157 embryonic development, myogenesis or for the development of the cardiovascular system

158 (26). No anatomic and behavioral defects were observed at birth. However, mice lacking 159 synemin developed a myopathic phenotype, possibly due to its role in linking desmin 160 filaments to costameres and sarcomeres $(12,26)$ and presented a reduced bone mass which 161 underlines that synemin plays also a role in bone homeostasis $(30,42)$. In muscle, synemin 162 inactivation leads to a decrease in maximal force, fatigue resistance and alteration of 163 membrane integrity associated with an occurrence of focal muscle degeneration and 164 regeneration. Synemin may play this role through its demonstrated binding to costameric and 165 sarcolemmal proteins, such as $\alpha$-actinin, vinculin $(1,44)$ and members of the dystroglycan 166 complex (29). In desmin KO mice, synemin no longer associates with Z-discs but remains 167 present in myotendinous and neuromuscular junctions, suggesting that synemin association 168 with junctional complexes, but not with Z-discs, is desmin-independent (6). This phenomenon 169 could be due to differential synemin isoform distribution since synemin $\mathrm{H}$ preferentially 170 localizes in cell-cell junctions while synemin M predominates in Z-discs (27). Moreover, 171 synemin $\mathrm{H}$ localizes to the M-band of the sarcomere through interaction with the M10 region 172 of titin (38). Synemin also affects the behavior of satellite cells. The balance between self173 renewal and differentiation of muscle satellite cells might be compromised in KO mice (26).

174 The authors suggested that synemin might influence satellite cell behavior through its role in 175 the regulation of PKA activity, which is important for myogenesis.

176 Loss of synemin also leads to cardiomyopathy. As described by Garcia-Pelagio et al. (11), the 177 genetic silencing of synemin in mice causes left ventricular systolic dysfunction as early as 178 the age of 3 months old. Although contractile and costameric proteins remained unchanged in 179 the old synemin $\mathrm{KO}$ hearts, some alterations were identified in several signaling proteins 
180 (PKA-RII, ERK and p70S6K) critical to cardiomyocyte function. The mice also present a left

181 ventricular hypertrophy and eventual dilation over time. Alterations in calcium handling

182 indicate that abnormalities originate specifically from cardiomyocytes and not from other

183 cardiac cells (11).

\section{Synemin-related disorders}

185 Synemin has also been linked to human diseases including heart disease, cancer, and 186 neurodegenerative disorders $(21,23,31,35,37)$. Its expression is upregulated in pathological 187 conditions such as neurotrauma, Alexandre disease and myofibrillar myopathies $(9,35)$. The 188 complete sequence of the human SYNM gene was reported by Titeux et al. and Mizuno et al. 189 in $2001(29,48)$. As a result of their studies, analyses were performed based on the full 190 transcript of the SYNM gene (transcript ID: ENST00000336292) in an effort to list all protein 191 changing variations (missense, frameshifts, nonsense). The Human Gene Mutation Database 192 and Clin Var database report the disease-causing mutations over the SYNM gene (see Table 1).

193 In this review, we analyzed the data concerning seven cases with skeletal myopathy or 194 cardiomyopathy associated with a variation in SYNM: Variant c.173C > T (p.A58V) (56); 195 variant c.598C $>$ T (p.Q200*) (28); variant c.815C $>$ T (p.A272V) (28); variant c. 1612T $>C$ 196 (p.W538R) (55); variant c.2356G>A (p.V746M) (54); variant c.2456_2457delAG (p.E819fs)

197 (51); variant c.4555C>T (p.Q1519*) (51) (see Table 2).

198 Synemin variants leading to skeletal myopathy: First, Mizuno et al. (28) suggested that 199 synemin could be a new candidate gene for myopathies. In that study, SYNM was screened for 200 mutations in 71 patients with various forms of myopathy for which a genetic cause could not 201 be identified. The authors reported 9 single-nucleotide polymorphisms (SNPs) in SYNM that 202 do not change the amino acid sequence and 12 SNPs that do. Nine silent SNPs, which did not 203 modify the amino acid sequence, were also identified in the patient population. Among twelve 204 modifier SNPs, 10 SNPs are common but the remaining 2 are rare. One of these rare SNPs 
creates a premature stop codon in the first exon of SYNM and was present in a patient with nemaline myopathy (NCBI36/Ensembl54: chr15:97463527, exon 1, c.598C >T, p.Q200*, non-conservative). This SNP was detected in the unaffected father of the patient. Neither the mother nor any of the other patients were carriers of this genetic variation. This variant is currently cataloged in gnomAD_v2.1.1 database (rs5030689) as a rare stop-gain variant

210 presenting in 1/14494 African, 3/24992 Latino, 68/8400 Ashkenazi Jewish, 39/63602

211 European (non-Finnish), 5/5074 Other origins of alleles. PolyPhen2 and SIFT prediction 212 analysis did not yield any predictions. Mutation Taster predicted this variant as being "disease 213 causing".

214 Synemin variants leading to cardiomyopathies: In 2006, Zeller et al. (54) reported the first 215 SYNM mutation (c.2356G $>$ A, p.V746M) associated with dilated cardiomyopathy. Zeller et al. 216 features a simple and reliable protocol for mutation screening in 25 known and putative 217 candidate genes for familial cardiomyopathy by heteroduplex analysis (54). Among the 218 screened families, one family carried a synemin mutation. Two simultaneous missense 219 mutations were identified in the patient suffering from dilated cardiomyopathy, one in SYNM 220 (c.2356G>A, p.V746M) and the other in the VEGF receptor 1 (FLT1) (c.411G >C, p.R54S) 221 gene. Both mutations were also found in the patient's mother and daughter. The daughter 222 showed a borderline left ventricular ejection fraction with a normal left ventricular dimension 223 and was clinically asymptomatic. The mother had regular echocardiographic findings and was 224 also asymptomatic (54). This variant is currently cataloged in gnomAD_v2.1.1 database as a 225 rare missense variant (rs142594053). This variant has an overall minor allele frequency (MAF) 226 of 0.0010476 , meaning it is still a rare variant (gnomAD allele frequency $<0,5 \%$ ). It has been 227 reported that this mutation of SYNM and FLT1 is involved in the VEGF-PLC gamma 1 228 signaling pathway that controls cardiac contractility in the embryonic heart (39). Disturbance 
of both mechanical integrity and calcium homeostasis of the cardiomyocyte is known to cause

230 cardiomyopathy.

231 In 2016, two variants of synemin were reported in dilated cardiomyopathy patients 232 (c.2456_2457delAG, p.E819fs; c.4555C>T, p.Q1519*) by Ware et al. (51). This study shows 233 the identification of 26 distinct rare heterozygous truncating variants in eight genes. Of the 26 234 truncated variants, 2 SYNM variants were identified; one being a missense mutation that 235 creates a premature stop codon $\left(\mathrm{c} .4555 \mathrm{C}>\mathrm{T}, \mathrm{p} . \mathrm{Q} 1519^{*}\right)$ and the second being a frameshift 236 mutation (c.2456_2457delAG, p.E819fs). Mutation Taster predicted the variant c.4555C $>$ T 237 (p.Q1519*) as being "disease causing": it results in the loss of protein tail interacting with 238 Dystrophin (DMD) and Utrophin (UTRN).

239 Based on its tissue-specific expression profiles and the available data on KO mice phenotypes, 240 Zlotina et al (56) suggested SYNM as a candidate gene contributing to the Ulnar-mammary 241 syndrome-like phenotype, a group of rare congenital conditions that combine cardiac 242 pathology (structural defect or arrhythmic disorder) and limb abnormality. Employing whole243 exome sequencing and bioinformatics strategies, a novel heterozygous missense variant in 244 SYNM was identified (GRCh37/hg19: chr15:99645578, exon 1, c.173C >T, p.A58V, 245 conservative) (56). Further comprehensive functional studies are required to evaluate possible 246 involvement of SYNM in genesis of complex heart-limb pathology. That variant is currently 247 cataloged in the gnomAD_v2.1.1 database as a rare missense variant presented only in one 248 allele of African origin among 3938 African alleles (1/3938). No accounts of the same 249 variation have been found in other populations studied and accepted into the gnomAD 250 database to date. That variant, having no "rs" accession number assigned yet, presents a minor 251 allele frequency (MAF) of 0.00000811. When analyzed as a single event, PolyPhen-2 and 252 SIFT prediction algorithm analyses qualified this variant as "benign/neutral", respectively. 253 Mutation Taster predicted the variant as being "polymorphism". 
254 The study of Zhang and al. (55) concerns the investigation of a Chinese family of three

255 generations in which four patients had dilated cardiomyopathy. Employing whole-exome 256 sequencing and bioinformatics strategies, a novel heterozygous missense mutation of SYNM

257 gene was identified (GRCh37/hg19: chr15:99670180, exon 4, c.1612T >C; p.W538R) and co-

258 segregated in the affected family members (55). The heterozygous variant was detected in all

259 living patients in this family and all family members carrying the wild-type genotype were

260 healthy. The missense mutation resulting in a substitution of tryptophan by arginine is located

261 in the C-terminal domain of synemin, known as the region close to the encoding AKAP site

262 of synemin which could be crucial for kinase activity. Cross-species alignment analysis of

263 synemin amino acid sequences revealed that this mutated site was highly conserved in

264 mammals, which suggests that the modification of tryptophan at position 538 may affect the

265 structure of synemin. As we demonstrated in Figure 3 using in silico modeling, this missense

266 mutation may cause structural changes in the synemin protein (see Figure 3). This variant is

267 currently cataloged in gnomAD_v2.1.1 database as a rare missense variant (rs551837344)

268 presenting in 8/17520 alleles of East Asian origin and 4/110162 alleles of European (non-

269 Finnish) origin. This variant has a minor allele frequency -10-(MAF) of 0.0000491. (see table

270 1: in PolyPhen2, probably damaging; in SIFT, deleterious; in Mutation Taster, disease

271 causing).

272 In silico modeling the structure of synemin protein and characterization of 273 synemin variants

274 It has been known for several years that all IFs are $\alpha$-helix type fibrous proteins; that is, they 275 contain coiled-coil helices (43). An amino acid sequence of the rod domain can assume this 276 structure. It contains a succession of seven residue peptides (heptads) (a-b-c-d-e-f-g), where a 277 and d are usually apolar residues, and are normally buried inside the protein core (16). Four 278 segments, widely referred to as $1 \mathrm{~A}, 1 \mathrm{~B}, 2 \mathrm{~A}$, and $2 \mathrm{~B}$, are separated by linkers. Linker L1 
connects $1 \mathrm{~A}$ to $1 \mathrm{~B}$; linker $\mathrm{L} 2$ joins $2 \mathrm{~A}$ to $2 \mathrm{~B}$. These are interspersed by short linkers that

280 cannot form a coiled-coil sequence of the form (hydrophilic-apolar). Linker L12 joins the 281 segments 1 and $2(16)$

282 Table 1 describes the different types of changes (conservative, missense, or stop) for 24

283 SYNM variant. Among them, 9 variants lead to conservative amino acids, 12 missense amino

284 acids, 2 premature stops, and one is a deletion. Moreover, Table 1 and Figure 2 report the 285 position of the amino acid variants with potential pathogenicity. Variants A58V, Q200*, 286 A272V related to cardiomyopathy or skeletal myopathy are located in the rod domain, 287 characterized by interactions with desmin, vimentin, vinculin, $\alpha$-dystrobrevin, dystrophin and 288 utrophin. Variant W538R is found near the region 556 to 707 with $\alpha$-helices described by Russell et al. (40) while also potentially being anchored to PKA. The missense variant V746M is located in the tail region of the $\mathrm{M}$ and $\mathrm{H}$ synemin but not of the $\mathrm{L}$ synemin, which is shorter. The tail region from 909 to 1564 , which is known to interact with zyxin, talin,

292 vinculin, $\alpha$-actinin and dystrophin, includes the variant Q1519* that causes the integration of 293 a premature stop codon.

294 Helical Wheel analyses performed by Khanamiryan et al (24) as well as by Russell et al (40) 295 show that certain regions of synemin could form an amphipathic structure. The software 296 Helical Wheel Custom Images and Interactive Java Applet software were used to search for 297 potential $\alpha$-helices within the synemin protein and for modifications of amino acid 298 interactions between the variants. It can be hypothesized that mutations leading to a change of 299 charge, distance and interactions between the amino acids of the helix are deleterious and 300 modify an association with their partners. In order to support our hypothesis, the interactions 301 between amino-acid residues were examined using in silico modeling. The modeling of 302 mutated synemin for missense residue 538 shows that the replacement of a tryptophan neutral 303 apolar hydrophobic residue by a positive hydrophilic arginine residue causes the loss of the 
304 hydrophobic interaction between residue 538 and residue leucine 541 (W--L--L vs R L--L)

305 (Table 2, Figure 3A). For missense mutation V746M, a nonpolar hydrophobic residue valine 306 changed to a nonpolar hydrophobic residue methionine does not change the interaction.

307 However, although variant 272 is a conservative mutation, the change of a nonpolar

308 hydrophobic residue alanine residue with a nonpolar hydrophobic residue valine creates a new 309 interaction between residue 272 valine and residue 276 leucine: (I--L A vs I--L--V) (Table 2,

310 Figure 3B). By contrast, for the conservative mutation of A58V, a nonpolar hydrophobic

311 residue alanine to a nonpolar hydrophobic residue valine does not modify the interactions

312 (Table 2, Figure 3B). It is evident that the change of hydrophobic interaction between amino 313 acids in the helix depends not only on the characteristic of the mutant residue, but also on the 314 property of its partner residue.

315 Concluding remarks: Pathogenic mutations or uncertain significant 316 variants?

317 To date, among the variants described of the SYNM gene, 7 could be associated in human to a 318 cardiomyopathy or a skeletal myopathy. Data on synemin KO mice suggest that synemin 319 plays an important role in skeletal muscle and in the heart. Synemin is thought to 320 mechanically stabilize the cardiomyocyte by linking the extracellular matrix to the Z-disc. 321 Synemin interacts with type III IFs desmin and vimentin, through the head and rod domain, 322 and interacts with membrane proteins through its long C-terminal tail. Furthermore, through 323 its interaction with cytoskeletal proteins dystrophin, utrophin and vinculin, synemin is able to 324 link those heteropolymers to adherent-type junctions within striated muscle cells, suggesting 325 that it plays an important role in the component assembly of the cytoskeleton and in 326 morphogenesis. In addition, we emphasize that in silico modeling of the helical salt-bridge 327 between residues could evaluate the impact of synemin on network organization or integration 328 into desmin filaments and be crucial to its interaction with cytoskeletal proteins. Two 
mutations in position 272 or in position 538, corresponding to patients with respectively

330 myopathy or cardiomyopathy, showed a change in neighbor amino acid interactions. The

331 position 538 near the encoding AKAP site of synemin could be crucial for kinase activity and

332 synemin-desmin heteropolymer filament assembly. Taken together, all data support a role for

333 synemin in the integrity of the intermediate filaments.

334 In this review, we summarized several studies indicate that a single mutant allele of SYNM

335 may cause disease as an autosomal dominant manner. In spite of the amount of accumulated

336 animal model research data and the information directly provided by medical genetic

337 diagnostic studies on human samples, we cannot correlate all SYNM variants related to

338 cardiomyopathy or skeletal myopathies. Like other rare variants, they may contribute to the

339 penetrance of potentially pathogenic structural or nucleotide variants of the genome, leading

340 to disease. Given that current state of exome data lacks the gene network analysis for

341 polymorphisms in myopathies, we propose a careful anlaysis and interpretation of additional

342 variants in SYNM and other related genes to be considered when evaluating a phenotype, both

343 in research and clinical context. Many studies including animal models, in vitro cell culture

344 assay, as well as in silico modeling of helical salt-bridges should be performed in order to

345 validate the clinical significance of SYNM variants.

346

347 


\section{Statement of author contributions}

349 ZX, YH performed in silico modeling of synemin protein; SK, DP, ZX contributed to genetic

350 variant analysis; DP, OA, ZL and ZX wrote the manuscript.

351

352 Fundings

353 Financial support has been provided by Sorbonne Université, CNRS, INSERM, the AFM-

354 Téléthon [contract numbers: 21833 and 22142] and the Fédération Française de Cardiologie.

355 Y.H. was supported by a fellowship from the AFM-Téléthon.

356

357 Acknowledments

358 We thank Dr Serge Fermandjian for valuable suggestions, Pr Ali Ladram for helpful advices,

359 Dr Ara Parlakian for critical reading of the manuscript and Sareen Kardjian for linguistic

360 improvements. We express our thanks to Pr L.E.Thornell for the gift of immunolabeling of

361 synemin and desmin presented in Figure 1.

362

363

364

365

366

367 


\section{References}

369 1. Bellin RM, Huiatt TW, Critchley DR, Robson RM. Synemin may function to

370 directly link muscle cell intermediate filaments to both myofibrillar Z-lines and costameres. $J$

371 Biol Chem 276: 32330-32337, 2001.

372 2. Bellin RM, Sernett SW, Becker B, Ip W, Huiatt TW, Robson RM. Molecular

373 characteristics and interactions of the intermediate filament protein synemin. Interactions with

374 alpha-actinin may anchor synemin-containing heterofilaments. J Biol Chem 274: 29493$37529499,1999$.

376 3. Bhosle RC, Michele DE, Campbell KP, Li Z, Robson RM. Interactions of

377 intermediate filament protein synemin with dystrophin and utrophin. Biochem Biophys Res 378 Commun 346: 768-777, 2006.

379 4. Capetanaki Y, Papathanasiou S, Diokmetzidou A, Vatsellas G, Tsikitis M. 380 Desmin related disease: a matter of cell survival failure. Curr Opin Cell Biol 32: 113-120, 3812015.

382 5. Carlsson L, Fischer C, Sjöberg G, Robson RM, Sejersen T, Thornell L-E. 383 Cytoskeletal derangements in hereditary myopathy with a desmin L345P mutation. Acta 384 Neuropathol 104: 493-504, 2002.

385 6. Carlsson L, Li ZL, Paulin D, Price MG, Breckler J, Robson RM, Wiche G,

386 Thornell LE. Differences in the distribution of synemin, paranemin, and plectin in skeletal 387 muscles of wild-type and desmin knock-out mice. Histochem Cell Biol 114: 39-47, 2000.

388 7. Chourbagi O, Bruston F, Carinci M, Xue Z, Vicart P, Paulin D, Agbulut 0.

389 Desmin mutations in the terminal consensus motif prevent synemin-desmin heteropolymer 390 filament assembly. Exp Cell Res 317: 886-897, 2011.

391 8. Clemen CS, Herrmann H, Strelkov SV, Schröder R. Desminopathies: pathology 392 and mechanisms. Acta Neuropathol 125: 47-75, 2013. 
394 Bauer R, Thorweihe J-M, Wenzel D, Schlötzer-Schrehardt U, Rasche V, Krsmanovic P, 395 Katus HA, Rottbauer W, Just S, Müller OJ, Friedrich O, Meyer R, Herrmann H, 396 Schrickel JW, Schröder R. The toxic effect of R350P mutant desmin in striated muscle of 397 man and mouse. Acta Neuropathol 129: 297-315, 2015.

398 10. de Souza Martins SC, Agbulut O, Diguet N, Larcher J-C, Paulsen BS, Rehen SK, 399 Moura-Neto V, Paulin D, Li Z, Xue Z. Dynamic expression of synemin isoforms in mouse 400 embryonic stem cells and neural derivatives. BMC Cell Biol 12: 51, 2011.

401 11. García-Pelagio KP, Chen L, Joca HC, Ward C, Jonathan Lederer W, Bloch RJ. 402 Absence of synemin in mice causes structural and functional abnormalities in heart. $J$ Mol 403 Cell Cardiol 114: 354-363, 2018.

404 12. García-Pelagio KP, Muriel J, O’Neill A, Desmond PF, Lovering RM, Lund L, 405 Bond M, Bloch RJ. Myopathic changes in murine skeletal muscle lacking synemin. Am $J$ 406 Physiol, Cell Physiol 308: C448-462, 2015.

407 13. Godsel LM, Hobbs RP, Green KJ. Intermediate filament assembly: dynamics to 408 disease. Trends Cell Biol 18: 28-37, 2008.

409 14. Granger BL, Lazarides E. Synemin: a new high molecular weight protein associated 410 with desmin and vimentin filaments in muscle. Cell 22: 727-738, 1980.

411 15. Green KJ, Böhringer M, Gocken T, Jones JCR. Intermediate filament associated 412 proteins. Adv Protein Chem 70: 143-202, 2005.

413 16. Herrmann H, Aebi U. Intermediate filaments: molecular structure, assembly 414 mechanism, and integration into functionally distinct intracellular Scaffolds. Annu Rev 415 Biochem 73: 749-789, 2004.

416 17. Hijikata T, Nakamura A, Isokawa K, Imamura M, Yuasa K, Ishikawa R, 417 Kohama K, Takeda S, Yorifuji H. Plectin 1 links intermediate filaments to costameric 
sarcolemma through beta-synemin, alpha-dystrobrevin and actin. J Cell Sci 121: 2062-2074,

4192008.

420 18. Hirako Y, Yamakawa H, Tsujimura Y, Nishizawa Y, Okumura M, Usukura J,

421 Matsumoto H, Jackson KW, Owaribe K, Ohara O. Characterization of mammalian

422 synemin, an intermediate filament protein present in all four classes of muscle cells and some

423 neuroglial cells: co-localization and interaction with type III intermediate filament proteins

424 and keratins. Cell Tissue Res 313: 195-207, 2003.

425 19. Izmiryan A, Cheraud Y, Khanamiryan L, Leterrier JF, Federici T, Peltekian E,

426 Moura-Neto V, Paulin D, Li Z, Xue ZG. Different expression of synemin isoforms in glia

427 and neurons during nervous system development. Glia 54: 204-213, 2006.

428 20. Izmiryan A, Peltekian E, Federici T, Paulin D, Li ZL, Xue ZG. Synemin isoforms

429 in astroglial and neuronal cells from human central nervous system. Neurochem Res 35: 881-

$430 \quad 887,2010$.

431 21. Jing R, Pizzolato G, Robson RM, Gabbiani G, Skalli O. Intermediate filament

432 protein synemin is present in human reactive and malignant astrocytes and associates with 433 ruffled membranes in astrocytoma cells. Glia 50: 107-120, 2005.

434 22. Jing R, Wilhelmsson U, Goodwill W, Li L, Pan Y, Pekny M, Skalli O. Synemin is 435 expressed in reactive astrocytes in neurotrauma and interacts differentially with vimentin and 436 GFAP intermediate filament networks. J Cell Sci 120: 1267-1277, 2007.

437 23. Kapoor S. Synemin: an evolving role in tumor growth and progression. J Cachexia 438 Sarcopenia Muscle 5: 347-348, 2014.

439 24. Khanamiryan L, Li Z, Paulin D, Xue Z. Self-assembly incompetence of synemin is 440 related to the property of its head and rod domains. Biochemistry 47: 9531-9539, 2008.

441 25. Konieczny P, Fuchs P, Reipert S, Kunz WS, Zeöld A, Fischer I, Paulin D, 442 Schröder R, Wiche G. Myofiber integrity depends on desmin network targeting to Z-disks 
and costameres via distinct plectin isoforms. J Cell Biol 181: 667-681, 2008.

444 26. Li Z, Parlakian A, Coletti D, Alonso-Martin S, Hourdé C, Joanne P, Gao-Li J, 445 Blanc J, Ferry A, Paulin D, Xue Z, Agbulut O. Synemin acts as a regulator of signalling 446 molecules during skeletal muscle hypertrophy. J Cell Sci 127: 4589-4601, 2014.

447 27. Lund LM, Kerr JP, Lupinetti J, Zhang Y, Russell MA, Bloch RJ, Bond M. 448 Synemin isoforms differentially organize cell junctions and desmin filaments in neonatal 449 cardiomyocytes. FASEB J 26: 137-148, 2012.

450 28. Mizuno Y, Puca AA, O’Brien KF, Beggs AH, Kunkel LM. Genomic organization 451 and single-nucleotide polymorphism map of desmuslin, a novel intermediate filament protein 452 on chromosome 15q26.3. BMC Genet 2: 8, 2001.

453 29. Mizuno Y, Thompson TG, Guyon JR, Lidov HG, Brosius M, Imamura M, Ozawa 454 E, Watkins SC, Kunkel LM. Desmuslin, an intermediate filament protein that interacts with 455 alpha -dystrobrevin and desmin. Proc Natl Acad Sci USA 98: 6156-6161, 2001.

456 30. Moorer MC, Buo AM, Garcia-Pelagio KP, Stains JP, Bloch RJ. Deficiency of the 457 intermediate filament synemin reduces bone mass in vivo. Am J Physiol, Cell Physiol 311: 458 C839-C845, 2016.

459 31. Noetzel E, Rose M, Sevinc E, Hilgers R-D, Hartmann A, Naami A, Knüchel R, 460 Dahl E. Intermediate filament dynamics and breast cancer: aberrant promoter methylation of 461 the Synemin gene is associated with early tumor relapse. Oncogene 29: 4814-4825, 2010.

462 32. Olivé M, Goldfarb L, Dagvadorj A, Sambuughin N, Paulin D, Li Z, Goudeau B, 463 Vicart P, Ferrer I. Expression of the intermediate filament protein synemin in myofibrillar 464 myopathies and other muscle diseases. Acta Neuropathol 106: 1-7, 2003.

465 33. Paul M, Skalli O. Synemin: Molecular Features and the Use of Proximity Ligation 466 Assay to Study Its Interactions. Meth Enzymol 568: 537-555, 2016.

467 34. Paulin D, Huet A, Khanamyrian L, Xue Z. Desminopathies in muscle disease. $J$ 
Pathol 204: 418-427, 2004.

469 35. Pekny T, Faiz M, Wilhelmsson U, Curtis MA, Matej R, Skalli O, Pekny M. 470 Synemin is expressed in reactive astrocytes and Rosenthal fibers in Alexander disease. 471 APMIS 122: 76-80, 2014.

472 36. Pitre A, Davis N, Paul M, Orr AW, Skalli O. Synemin promotes AKT-dependent 473 glioblastoma cell proliferation by antagonizing PP2A. Mol Biol Cell 23: 1243-1253, 2012.

474 37. Poot M, Verrijn Stuart AA, van Daalen E, van Iperen A, van Binsbergen E, 475 Hochstenbach R. Variable behavioural phenotypes of patients with monosomies of $15 \mathrm{q} 26$ 476 and a review of 16 cases. Eur J Med Genet 56: 346-350, 2013.

477 38. Prudner BC, Roy PS, Damron DS, Russell MA. $\alpha$-Synemin localizes to the M-band 478 of the sarcomere through interaction with the M10 region of titin. FEBS Lett 588: 4625-4630, 4792014.

480 39. Rottbauer W, Just S, Wessels G, Trano N, Most P, Katus HA, Fishman MC. 481 VEGF-PLCgamma1 pathway controls cardiac contractility in the embryonic heart. Genes Dev 482 19: 1624-1634, 2005.

483 40. Russell MA, Lund LM, Haber R, McKeegan K, Cianciola N, Bond M. The 484 intermediate filament protein, synemin, is an AKAP in the heart. Arch Biochem Biophys 456: $485 \quad 204-215,2006$.

486 41. Schmitt-Graeff A, Jing R, Nitschke R, Desmoulière A, Skalli O. Synemin 487 expression is widespread in liver fibrosis and is induced in proliferating and malignant biliary 488 epithelial cells. Hum Pathol 37: 1200-1210, 2006.

489 42. Skalli O. The cytoskeleton meets the skeleton. Focus on "Deficiency of the 490 intermediate filament synemin reduces bone mass in vivo." Am J Physiol, Cell Physiol 311: $491 \quad$ C837-C838, 2016.

492 43. Steinert PM, Zimmerman SB, Starger JM, Goldman RD. Ten-nanometer filaments 
493 of hamster BHK-21 cells and epidermal keratin filaments have similar structures. Proc Natl

494 Acad Sci USA 75: 6098-6101, 1978.

495 44. Sun N, Critchley DR, Paulin D, Li Z, Robson RM. Human alpha-synemin interacts 496 directly with vinculin and metavinculin. Biochem J 409: 657-667, 2008.

497 45. Sun N, Huiatt TW, Paulin D, Li Z, Robson RM. Synemin interacts with the LIM 498 domain protein zyxin and is essential for cell adhesion and migration. Exp Cell Res 316: 491$499505,2010$.

500 46. Swärd K, Krawczyk KK, Morén B, Zhu B, Matic L, Holmberg J, Hedin U, 501 Uvelius B, Stenkula K, Rippe C. Identification of the intermediate filament protein 502 synemin/SYNM as a target of myocardin family coactivators. Am. J. Physiol., Cell Physiol. 503 317: C1128-C1142, 2019.

504 47. Tawk M, Titeux M, Fallet C, Li Z, Daumas-Duport C, Cavalcante LA, Paulin D, 505 Moura-Neto V. Synemin expression in developing normal and pathological human retina and 506 lens. Exp Neurol 183: 499-507, 2003.

507 48. Titeux M, Brocheriou V, Xue Z, Gao J, Pellissier JF, Guicheney P, Paulin D, Li Z. 508 Human synemin gene generates splice variants encoding two distinct intermediate filament 509 proteins. Eur J Biochem 268: 6435-6449, 2001.

510 49. Tsikitis M, Galata Z, Mavroidis M, Psarras S, Capetanaki Y. Intermediate 511 filaments in cardiomyopathy. Biophys Rev 10: 1007-1031, 2018.

512 50. Uyama N, Zhao L, Van Rossen E, Hirako Y, Reynaert H, Adams DH, Xue Z, Li

513 Z, Robson R, Pekny M, Geerts A. Hepatic stellate cells express synemin, a protein bridging 514 intermediate filaments to focal adhesions. Gut 55: 1276-1289, 2006.

515 51. Ware JS, Li J, Mazaika E, Yasso CM, DeSouza T, Cappola TP, Tsai EJ, Hilfiker516 Kleiner D, Kamiya CA, Mazzarotto F, Cook SA, Halder I, Prasad SK, Pisarcik J, 517 Hanley-Yanez K, Alharethi R, Damp J, Hsich E, Elkayam U, Sheppard R, Kealey A, 
Alexis J, Ramani G, Safirstein J, Boehmer J, Pauly DF, Wittstein IS, Thohan V, Zucker

MJ, Liu P, Gorcsan J, McNamara DM, Seidman CE, Seidman JG, Arany Z, IMAC-2

and IPAC Investigators. Shared Genetic Predisposition in Peripartum and Dilated

521 Cardiomyopathies. N Engl J Med 374: 233-241, 2016.

522 52. Winter DL, Paulin D, Mericskay M, Li Z. Posttranslational modifications of desmin

523 and their implication in biological processes and pathologies. Histochem Cell Biol 141: 1-16, 5242014.

525 53. Xue ZG, Cheraud Y, Brocheriou V, Izmiryan A, Titeux M, Paulin D, Li Z. The

526 mouse synemin gene encodes three intermediate filament proteins generated by alternative 527 exon usage and different open reading frames. Exp Cell Res 298: 431-444, 2004.

528 54. Zeller R, Ivandic BT, Ehlermann P, Mücke O, Zugck C, Remppis A, Giannitsis E, 529 Katus HA, Weichenhan D. Large-scale mutation screening in patients with dilated or 530 hypertrophic cardiomyopathy: a pilot study using DGGE. J Mol Med 84: 682-691, 2006.

531 55. Zhang S-B, Liu Y-X, Fan L-L, Huang H, Li J-J, Jin J-Y, Xiang R. A novel 532 heterozygous variant p.(Trp538Arg) of SYNM is identified by whole-exome sequencing in a 533 Chinese family with dilated cardiomyopathy. Ann Hum Genet 83: 95-99, 2019.

534 56. Zlotina A, Kiselev A, Sergushichev A, Parmon E, Kostareva A. Rare Case of 535 Ulnar-Mammary-Like Syndrome With Left Ventricular Tachycardia and Lack of TBX3 536 Mutation. Front Genet 9: 209, 2018. 


\section{Figure Legends}

544 Figure 1. Schematic representation of the human synemin gene (SYNM) and protein

545 isoforms (SYNM). (A) Organization of synemin gene. The SYNM encodes three RNA

546 corresponding respectively to $\mathrm{M}$ (exons 1-2-3-4-5), H (exons 1-2-3-4a) and L (exons 1-2-3-5)

547 through an alternative splicing mechanism, giving rise to three proteins. (B) Organisation of

548 synemin isoforms. High $(\mathrm{H})$ or $\alpha$-synemin $(170 \mathrm{kDa})$, Middle $(\mathrm{M})$ or $\beta$-synemin $(140 \mathrm{kDa})$,

549 and Low (L) synemin (41 kDa) with the same head (10 amino acids) and rod domains (312

550 amino acid) but with different lengths of the C-terminal end (respectively 1243, 931 and 6

551 amino-acids). AA: amino acid. (C) Immunofluorescence detection of desmin (green) and

552 synemin (red) in human skeletal muscle (from Lars-Eric Thornell, Umeå University, Sweden).

553 Synemin co-localizes with desmin at the myofibrillar Z-lines. Bar: $5 \mu \mathrm{m}$.

554

555 Figure 2. Localization of synemin interacting regions with its partners. Tripartite structure

556 of synemin comprising a central $\alpha$-helical rod domain (amino acid position from 11 to 322)

557 flanked by non-helical head (amino acid position from 1 to 10) and tail domains (amino acid

558 position from 323 to 1565 ). Note that the amino acids 1151 to 1463 are absent of $\mathrm{M}$ synemin

559 (48). Protein interactions with parts of rod domain of synemin are presented: Desmin (7),

560 Vimentin (3), Vinculin (44), $\alpha$-Dystrobrevin (29), Dystrophin (3), Utrophin (3) interacting

561 site. Interactions with parts of the tail domain of M and H synemin (from 909 to 1564 amino

562 acid): Zyxin (45), Talin (44), Vinculin (44), $\alpha$-Actinin (2), Dystrophin (3). Triangles show the

563 positions of 7 variants examined in this review; numbers correspond to the position of

564 mutated amino acids.

565 
566 Figure 3. Schematic representation of helical part of synemin variants: Computer analyses

567 (DNASTAR) were used to search for potential $\alpha$-helices and the modifications of amino acid

568 interactions in the variants. (A) The modeling of mutated synemin for residue 538 shows that

569 the replacement of a neutral apolar hydrophobic tryptophan residue by a positive hydrophilic

570 arginine residue causes a suppression of the hydrophobic interactions between residue 538

571 and leucine 541. (B) Schematic presentation of interactions for mutants: (A58V) conservative

572 without modification, (A272V) with a new hydrophobic bond, (W538R) missense with loss

573 of the hydrophobic bond or (V746M) without modification.

574

575

576

577 
Table 1. SYNM polymorphism: variants, prediction and functional consequences of selected 24 SYNM variants.

\begin{tabular}{|c|c|c|c|c|c|c|c|c|c|c|}
\hline 1 & 2 & 3 & 4 & 5 & 6 & 7 & 8 & 9 & 10 & 11 \\
\hline $\begin{array}{c}\text { Genomic } \\
\text { Coordinates }\end{array}$ & $\begin{array}{l}\text { Codon } \\
\text { Change }\end{array}$ & $\begin{array}{c}\text { Base } \\
\text { Variant }\end{array}$ & $\begin{array}{c}\text { Amino acid } \\
\text { change }\end{array}$ & Effect & $\begin{array}{c}\text { Phenotype } \\
\text { (Associated Disease) }\end{array}$ & References & $\begin{array}{c}\text { SIFT } \\
\text { prediction }\end{array}$ & $\begin{array}{l}\text { PolyPhen } 2 \\
\text { prediction }\end{array}$ & $\begin{array}{l}\text { Mutation taster } \\
\text { prediction }\end{array}$ & \begin{tabular}{|c} 
dbSNP \\
accession nr.
\end{tabular} \\
\hline 99645578 & CAG G[C/T]C CGC & $173 C>T$ & A58V & c & $\begin{array}{l}\text { Ulnar-Mammary-Like } \\
\text { syndrome, } \\
\text { Cardiomyopathy }\end{array}$ & (56) & damaging & $\begin{array}{l}\text { benign / possibly } \\
\text { damaging }\end{array}$ & polymorphism & rs 1367107502 \\
\hline 99646003 & GTG [C/T]AG CTG & $598 \mathrm{C}>\mathrm{T}$ & Q200* & Stop & Nemaline Myopathy & (28) & $\mathrm{n} / \mathrm{a}$ & $n / a$ & disease causing & rs5030689 \\
\hline 99653800 & AGA G[T/C]G ATT & $815 C>T$ & $\mathrm{~A} 272 \mathrm{~V}$ & C & nd & (28) & tolerated & benign & polymorphism & rs 2305445 \\
\hline 99666979 & CAC [G/A]TT GAA & $988 \mathrm{G}>\mathrm{A}$ & V330I & C & nd & (28) & tolerated & benign & polymorphism & rs5030691 \\
\hline 99669628 & GAA $[\mathrm{A} / \mathrm{T}] \mathrm{GG}$ AAT & $1063 A>T$ & R335W & $M$ & nd & (28) & damaging & possibly damaging & polymorphism & rs3743242 \\
\hline 99669628 & GAA $[\mathrm{A} / \mathrm{T}] \mathrm{GG}$ AAT & $1065 \mathrm{~T}>\mathrm{A}$ & W355R & $M$ & nd & unpublished & damaging & possibly damaging & polymorphism & rs3743242 \\
\hline 99669684 & TCG GC[A/G] CTG & $1119 \mathrm{~T}>\mathrm{C}$ & V373A & $\mathrm{C}$ & nd & unpublished & tolerated & $n / a$ & polymorphism & rs1555485392 \\
\hline 99670180 & AGA [T/C]GG GAA & $1612 T>C$ & W538R & $\mathrm{M}$ & Cardiomyopathy & (55) & damaging & damaging & disease & rs551837344 \\
\hline 99670265 & TCA C[C/T]G AAG & $1700 C>T$ & P567L & C & nd & (28) & tolerated & benign & polymorphism & rs3743244 \\
\hline 99670400 & GCA G[A/C]A GCA & $1835 \mathrm{~A}>\mathrm{C}$ & E612A & $M$ & nd & (28) & damaging & benign & polymorphism & rs5030692 \\
\hline 99670624 & ATA [G/C]חT GTG & $2061 C>G$ & L687V & C & nd & unpublished & tolerated & benign & polymorphism & - \\
\hline 99670725 & GGC AA[G/C] TCA & $2160 G>C$ & K720N & c & nd & unpublished & damaging & $\begin{array}{c}\text { benign / possibly } \\
\text { damaging }\end{array}$ & polymorphism & - \\
\hline 99670801 & AAC [G/A]TG GAG & $2356 \mathrm{G}>\mathrm{A}$ & V746M & $M$ & Cardiomyopathy & (54) & damaging & probably damaging & disease causing & rs142594053 \\
\hline 99670847 & AAG C[C/T]G GAG & $2282 C>T$ & P761L & C & nd & (28) & tolerated & benign & polymorphism & rs3743247 \\
\hline 99671012 & GTG G[A/G]A GAA & $2445 A>G$ & E815G & $M$ & nd & unpublished & damaging & probably damaging & disease causing & - \\
\hline 99671022-23 & N/A & 2456_2457delAG & E819fs & FS & Cardiomyopathy & (51) & $n / a$ & $n / a$ & disease causing & rs 1555485680 \\
\hline 99671401 & $\mathrm{CCC}[\mathrm{C} / \mathrm{T}] \mathrm{GG} \mathrm{CAG}$ & $2836 C>T$ & R946W & $\mathrm{M}$ & nd & (28) & damaging & possibly damaging & polymorphism & rs5030694 \\
\hline 99671492 & GGG C[A/G]G GGT & $2927 A>G$ & Q976R & $M$ & nd & $(28)$ & tolerated & benign & polymorphism & rs5030695 \\
\hline 99671741 & GCC C[C/T $] \mathrm{G} \mathrm{GCT}$ & $3176 C>T$ & P1059L & $c$ & nd & (28) & damaging & benign & polymorphism & rs5030697 \\
\hline 99671765 & AGG C[G/C]T TGG & $3200 G>C$ & R1067P & $\mathrm{M}$ & nd & $(28)$ & damaging & benign & polymorphism & rs5030698 \\
\hline 99671795 & СCT T[C/T]A GGC & $3230 C>T$ & S1077L & $M$ & nd & (28) & damaging & benign & polymorphism & rs5030699 \\
\hline 99672599 & CAC G[G/C]A GAG & $4032 G>C$ & G1345A & $M$ & nd & unpublished & tolerated & benign & polymorphism & rs7167599 \\
\hline 99672722 & GCA G[A/G]G GAC & $4155 \mathrm{~A}>\mathrm{G}$ & E1386G & $M$ & nd & unpublished & tolerated & benign & polymorphism & rs2292288 \\
\hline 99673120 & GAA $[\mathrm{C} / \mathrm{T}] \mathrm{AG}$ GGC & $4555 C>T$ & Q1519* & Stop & Cardiomyopathy & (51) & $n / a$ & $n / a$ & disease causing & - \\
\hline
\end{tabular}

Columns 1, 2, 11, data from NCBI; https://www.ncbi.nlm.nih.gov/projects/sviewer. Column 1 indicates genomic coordinates; column 2 indicates codon change; and column 11 indicates db SNP (single nucleotide polymorphism) accession numbers of selected 24 SYNM variants. Column 3 indicates base variant; and column 4 indicates amino acid change from published $(28,51,54-56)$ and unpublished data ( $\mathrm{Z} \mathrm{Li}$, Sorbonne Université, France). For amino acid changes analyses, the sequence of synemin from Titeux et al (48) were used (GenBank under accession numbers AJ310522 for the $\alpha$ isoform (Synemin H) and AJ310521 for the $\beta$ isoform (Synemin M). Column 5 indicates effect of mutations: (C) Conservative, (M) Missense, (FS) Frame Shift, Stop. Column 6 indicates the phenotype or associated disease from published data $(28,51,54-56)$. nd: non-determined. Column 8 to 10 indicate prediction analyses; SIFT prediction: consequences of mutations: damaging, tolerated, n/a: not available; PolyPhen2 prediction: consequence of mutations for synemin protein NP_663780 (isoform $\alpha / \mathrm{H}$ ) / NP_056101 (isoform $\beta / \mathrm{M}$ ); benign, probably damaging, possibly damaging; $\overline{\text { Mutation }}$ taster prediction: consequence of mutations polymorphism or disease causing. Gray rows indicate the seven variants selected in this review. 
Table 2. Synemin variants from patients with skeletal myopathy or cardiomyopathy.

\begin{tabular}{|c|c|c|c|c|c|}
\hline Location & $\begin{array}{c}\text { Amino Acid } \\
\text { Change }\end{array}$ & Mutation Site & $\begin{array}{l}\text { Changes in Amino } \\
\text { Acid Interaction } \\
\text { (wild-type/mutant) }\end{array}$ & Modification & $\begin{array}{l}\text { Associated } \\
\text { Disease } \\
\text { (References) }\end{array}$ \\
\hline Exon 1 & Conservative & 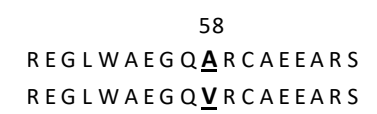 & $A A L / V A L$ & No modification & $\begin{array}{c}\text { Cardiomyopathy } \\
\text { (56) }\end{array}$ \\
\hline Exon 1 & Stop & $\begin{array}{l}200 \\
\text { VAESWRETV } \underline{\mathbf{Q}} \text { LYEDEVRE } \\
\text { VAESWRETV } \underline{\underline{*}}\end{array}$ & $n / a$ & - & $\begin{array}{l}\text { Nemaline } \\
\text { Myopathy } \\
\text { (28) }\end{array}$ \\
\hline Exon 2 & Conservative & $\begin{array}{c}272 \\
\text { GIQAEERQR } \underline{\mathbf{A} I D C L E D E K} \\
\text { GIQAEERQR } \underline{\mathbf{V}} \text { IDCLEDEK }\end{array}$ & I--LA / I--L--V & $\begin{array}{l}\text { New hydrophobic } \\
\text { bond creation }\end{array}$ & $\begin{array}{l}\text { nd } \\
(28)\end{array}$ \\
\hline Exon 4 & Missense & $\begin{array}{l}538 \\
\text { KASEERNLR } \underline{\mathbf{W}} \text { EELTKLDK } \\
\text { KASEERNLR } \underline{\mathbf{R} E E L T K L D K}\end{array}$ & W--L--L / R L--L & $\begin{array}{c}\text { Hydrophobic bond } \\
\text { lost }\end{array}$ & $\begin{array}{c}\text { Cardiomyopathy } \\
\text { (55) }\end{array}$ \\
\hline Exon 4 & Missense & 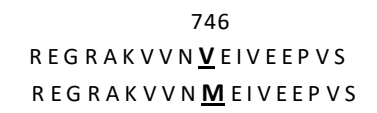 & V--V--V--V / V--M--V--V & No modification & $\begin{array}{c}\text { Cardiomyopathy } \\
\text { (54) }\end{array}$ \\
\hline Exon 4 & Frameshift & $\begin{array}{l}819 \\
\text { NTTHVEEVTE्EAGDSEGEQ } \\
\text { NTTHVEEVT } \underline{\mathbf{f}} \underline{\mathrm{s}}\end{array}$ & $n / a$ & - & $\begin{array}{c}\text { Cardiomyopathy } \\
\text { (51) }\end{array}$ \\
\hline Exon 5 & Stop & $\begin{array}{l}1519 \\
\text { GEGDQAHRE } \underline{\mathbf{Q} G K E Q A M F D} \\
\text { GEGDQAHRE } \stackrel{*}{*}\end{array}$ & $n / a$ & - & $\begin{array}{c}\text { Cardiomyopathy } \\
\text { (51) }\end{array}$ \\
\hline
\end{tabular}

Different SYNM variants and their consequences: A58V (Ulnar-mammary syndrome) (56), Q200* (Nemaline myopathy) (28), A272V (nd: no-determined) (28), W538R (Dilated cardiopathy) (55), V746M (Dilated cardiopathy) (54), E819fs (Cardiomyopathy) (51), Q1519* (Cardiomyopathy) (51). Different types of amino acid interaction changes are indicated for four variants: (A58V) conservative without modification, (A272V) with a new hydrophobic bond, (W538R) missense with loss of the hydrophobic bond or (V746M) without modification. (--) indicates hydrophobic interaction. n/a: no applicable (see figure 3). 
A

\section{Synemin Gene (SYNM)}

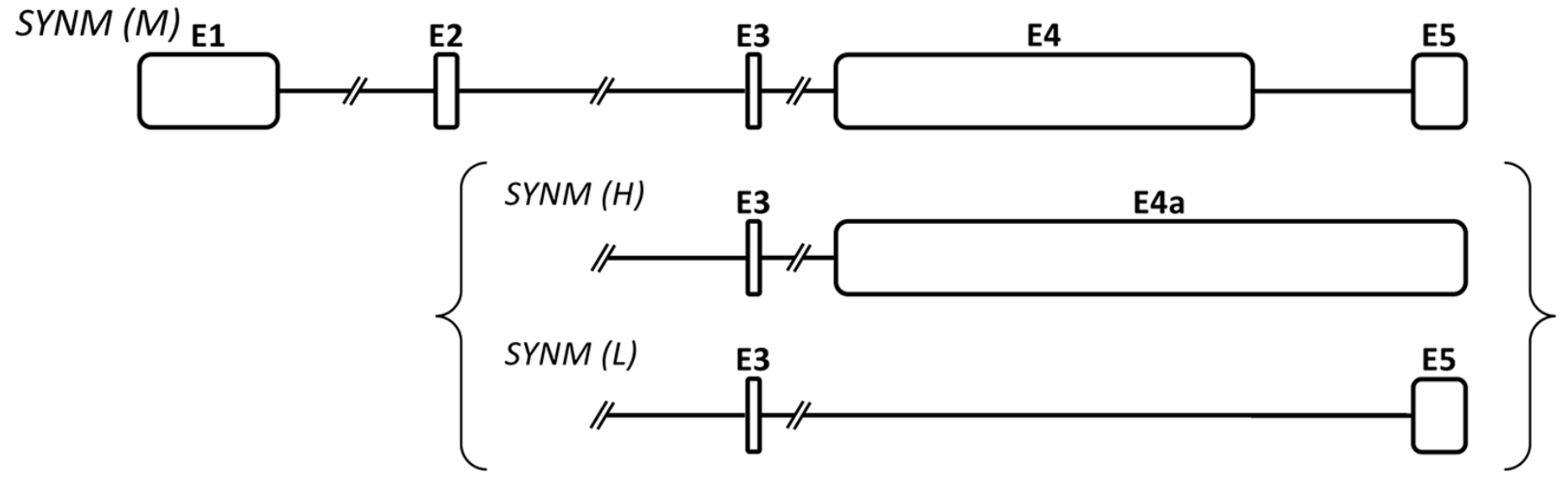

\section{Synemin Protein (SYNM)}

Head + Rod

Tail

322 AA
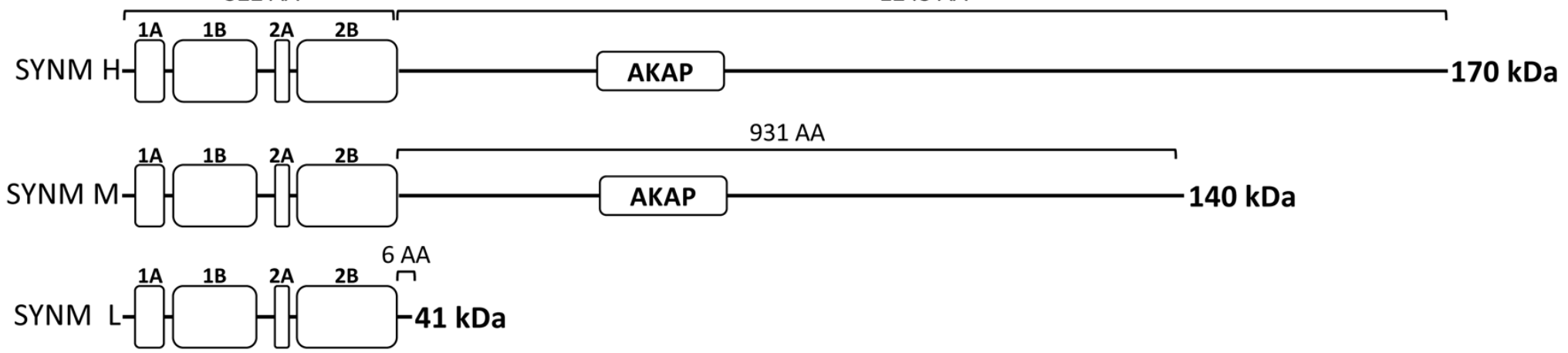

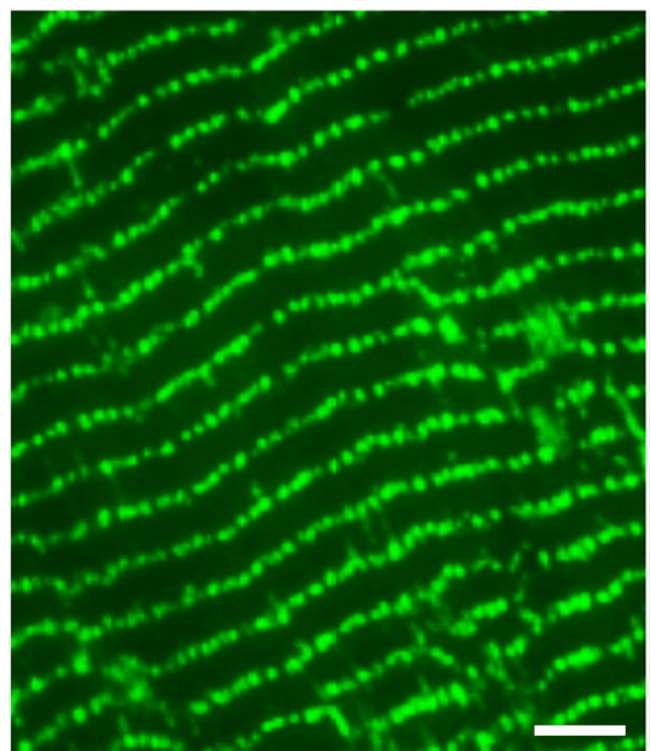

Desmin

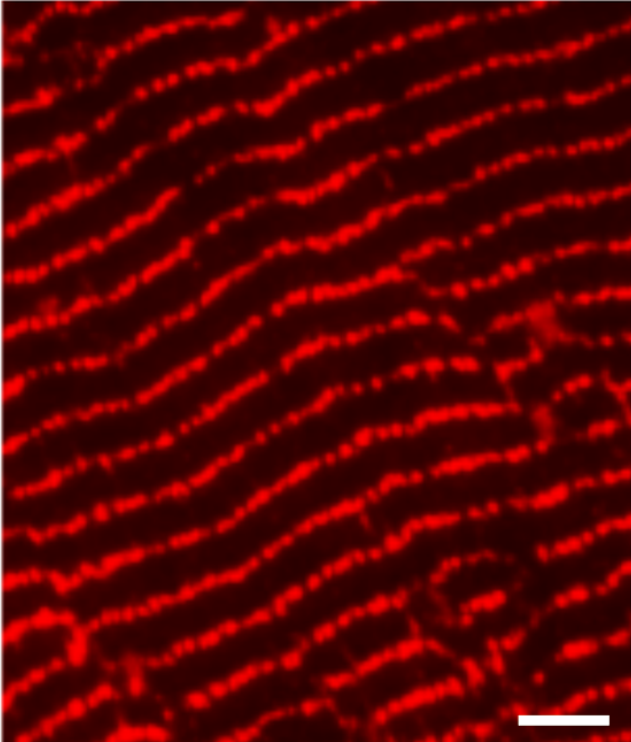

Synemin

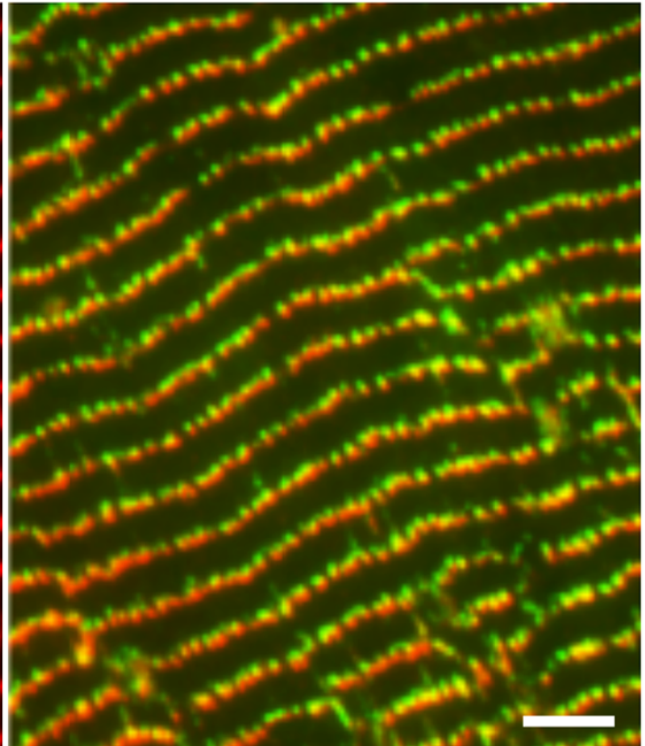

Merged 


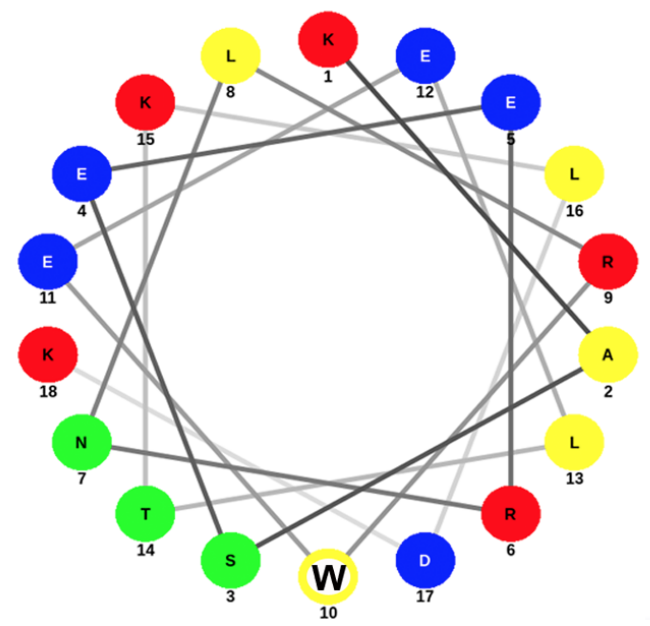

KASEERNLRWEELTKLDK WT

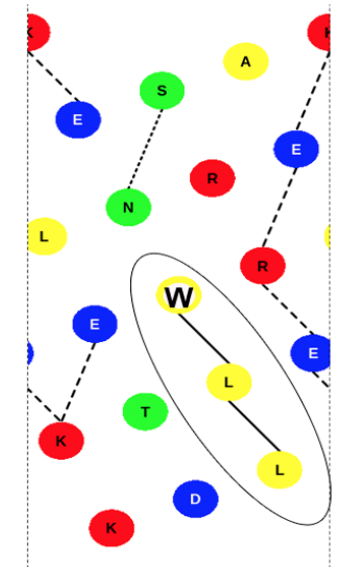

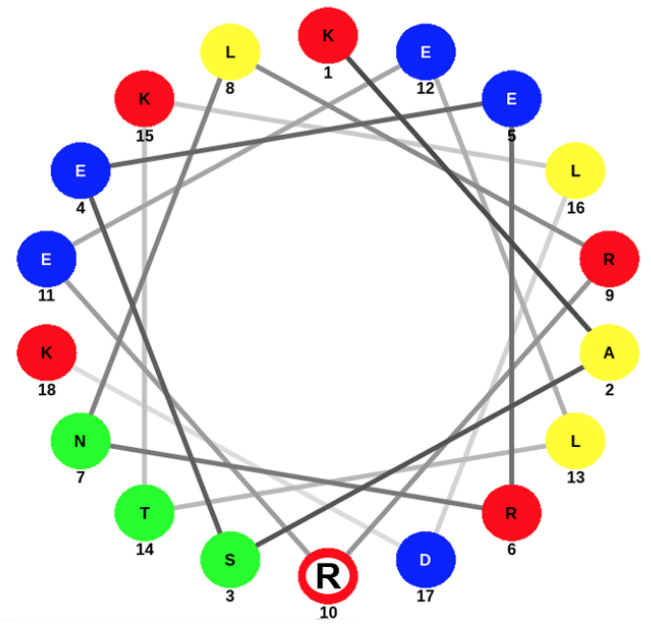

KASEERNLRREELTKLDK mutant

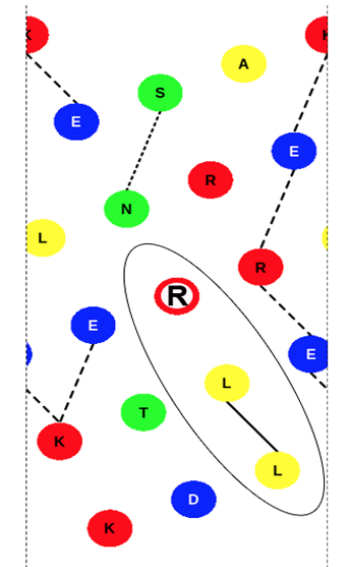

B<smiles>[CH]C#[Co]</smiles>

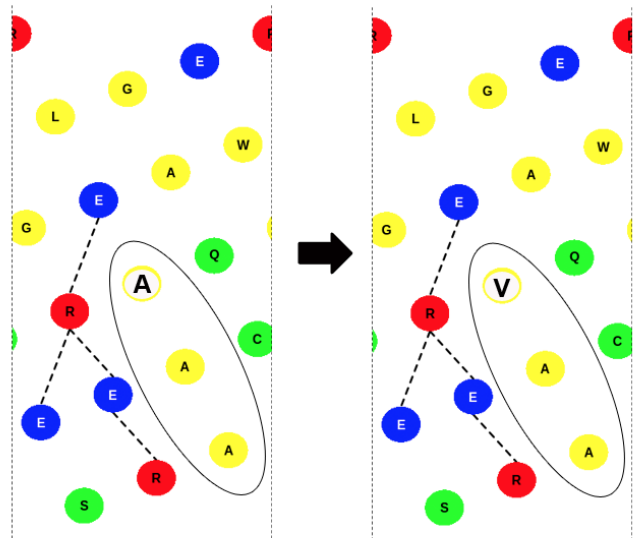

W538R
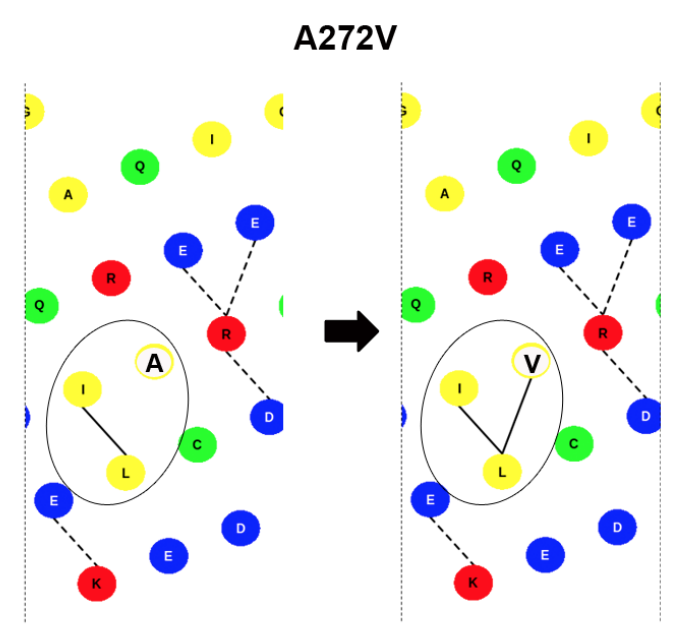

V746M
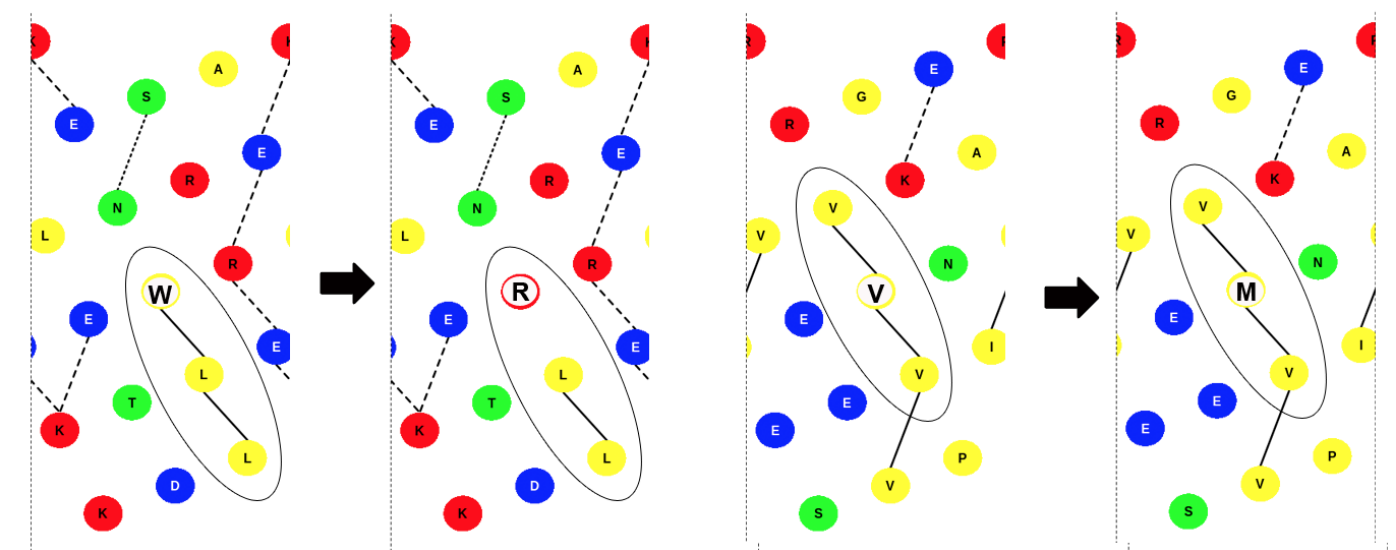